\title{
ACTIVIDAD SÍSMICA EN COSTA RICA DURANTE EL 2013
}

\author{
SEISMICITY IN COSTA RICA DURING 2013
}

\author{
Lepolt Linkimer*, Juan L. Porras, Rafael Barquero, María C. Araya, \\ Oscar H. Lücke, Magda Taylor \& Wilfredo Rojas
}
Red Sismológica Nacional (RSN: UCR-ICE), Apdo. 214-2060, San Pedro, Costa Rica
*Autor para contacto: lepolt.linkimer@ucr.ac.cr

(Recibido: 03/06/2014; aceptado: 06/10/2014)

\begin{abstract}
During 2013, the National Seismological Network (RSN: UCR-ICE) located 2547 earthquakes and reported 261 felt events. There were seven significant earthquakes with magnitude $\geq 5,0$. The majority ( $85 \%)$ of earthquakes were shallow $(<30 \mathrm{~km}$ ) and $56 \%$ had a low magnitude (Mw 3,0-3,9). Local and regional faulting caused $52 \%$ of the felt earthquakes and $45 \%$ occurred due to the subduction of the Cocos Plate beneath the Caribbean Plate and the Panama microplate. The highest intensity observed during 2013 was VI (Modified Mercalli, MM) in the Sixaola region (Limon) during the May 27 earthquake (5.6 Mw) with epicenter in western Panama.

Keywords: Seismology, RSN, seismicity in 2013, seismic sources, Costa Rica.

RESUMEN: Durante el 2013 la Red Sismológica Nacional (RSN: UCR-ICE) localizó 2547 sismos de los cuales 261 fueron sentidos por la población. Se percibieron en Costa Rica siete sismos con magnitud Mw $\geq 5,0$. La mayoría ( $85 \%)$ de los sismos percibidos fueron superficiales $(<30 \mathrm{~km})$ y el $56 \%$ tuvo una magnitud baja (Mw 3,0-3,9). El fallamiento local y regional provocó el $52 \%$ de los sismos, seguido por el proceso de subducción de la placa del Coco bajo la placa Caribe y la microplaca de Panamá que originó el 45\%. La intensidad máxima observada en el 2013 fue de VI (Mercalli Modificada, MM) para la zona de Sixaola (Limón) debido al sismo del 27 de mayo (Mw 5,6) con epicentro en el occidente de Panamá.

Palabras clave: Sismología, RSN, sismicidad del 2013, fuentes sísmicas, Costa Rica.
\end{abstract}




\section{INTRODUCCIÓN}

En el 2013 la Red Sismológica Nacional (RSN: UCR-ICE) localizó 2547 sismos de los cuales 261 fueron percibidos por la población (Figs 1 y 2, Cuadro 1). La cantidad de sismos sentidos en el año 2012 (264) fue muy similar a la del 2013, no obstante la cantidad de sismos localizados fue mucho mayor (3455). El 2013 es uno de los años con mayor cantidad de sismos sentidos en el registro de la RSN. Desde 1976, los años con mayor cantidad de sismos sentidos han sido 1990, 1991, 2012 y 2013, con 191, 353, 264 y 261, respectivamente. El promedio anual de sismos sentidos para el periodo 1976-2013 es de 88 .

La Red Sismológica Nacional (RSN) fue creada mediante un convenio entre la Sección de Sismología, Vulcanología y Exploración Geofísica de la Escuela Centroamericana de Geología de la Universidad de Costa Rica (UCR) y el Área de Amenazas y Auscultación Sísmica y Volcánica del Instituto Costarricense de Electricidad (ICE). La sede y centro de registro de la RSN se localiza en las instalaciones en la Escuela Centroamericana de Geología de la UCR y funciona desde 1973. Los sismos localizados durante el 2013, fueron registrados por una red de 62 estaciones sismológicas ubicadas en diferentes partes de Costa Rica, Panamá y Nicaragua. La detección y localización de los sismos localizados durante el 2013 se realizó a través de los sistemas Seiscomp, Earthworm y SeisAn. La lectura de arribos de ondas, localización y cálculo de magnitudes, se llevó a cabo mediante el programa HYP (Lienert \& Havskov, 1995) y la última versión del software sismológico SeisAn (Ottemöller et al., 2011). Para el proceso de localización se empleó un modelo de velocidades de la onda Primaria $(\mathrm{P})$ de siete capas, una razón de velocidades $(\mathrm{Vp} / \mathrm{Vs})$ de 1,74 y se calculó la magnitud momento (Mw) para cada sismo.

\section{CARACTERÍSTICAS DE LOS SISMOS SENTIDOS}

A continuación se describe la sismicidad sentida localizada durante el año 2013 tomando en cuenta su distribución temporal y por profundidad, magnitud (Mw) y origen.

\section{Distribución temporal}

El promedio mensual de sismos sentidos para el año 2013 fue de 22. Los meses con mayor cantidad de sismos sentidos fueron agosto y enero con 31 y 30, sismos respectivamente (Fig. 3A). Los meses con la menor cantidad de sismos sentidos fueron julio y septiembre, en los que se reportaron solamente nueve y 14 sismos, respectivamente. El resto de meses del año presentaron una cantidad de sismos que varió entre 17 y 25 (Fig. 3A).

\section{Distribución por magnitud (Mw)}

El sismo de mayor magnitud del 2013 ocurrió el 15 de junio al oeste de Managua en la costa pacífica de Nicaragua. Este sismo se ubicó a $164 \mathrm{~km}$ al noroeste de Peñas Blancas, tuvo una magnitud de 6,2 y fue sentido leve en la provincia de Guanacaste y en el Valle Central, Grecia y Zarcero. El sismo sentido de mayor magnitud ubicado dentro del territorio costarricense ocurrió el 5 de setiembre, con una magnitud de 5,9 y se ubicó 63 $\mathrm{km}$ al suroeste de Cabo Velas de Santa Cruz, Guanacaste. Debido a su lejanía con las zonas pobladas de Guanacaste este sismo fue percibido de forma leve. Este sismo se localizó $\sim 100 \mathrm{~km}$ al noroeste del epicentro del terremoto de Sámara que ocurrió exactamente un año antes, el 5 de setiembre del 2012 (Mw 7,6). 


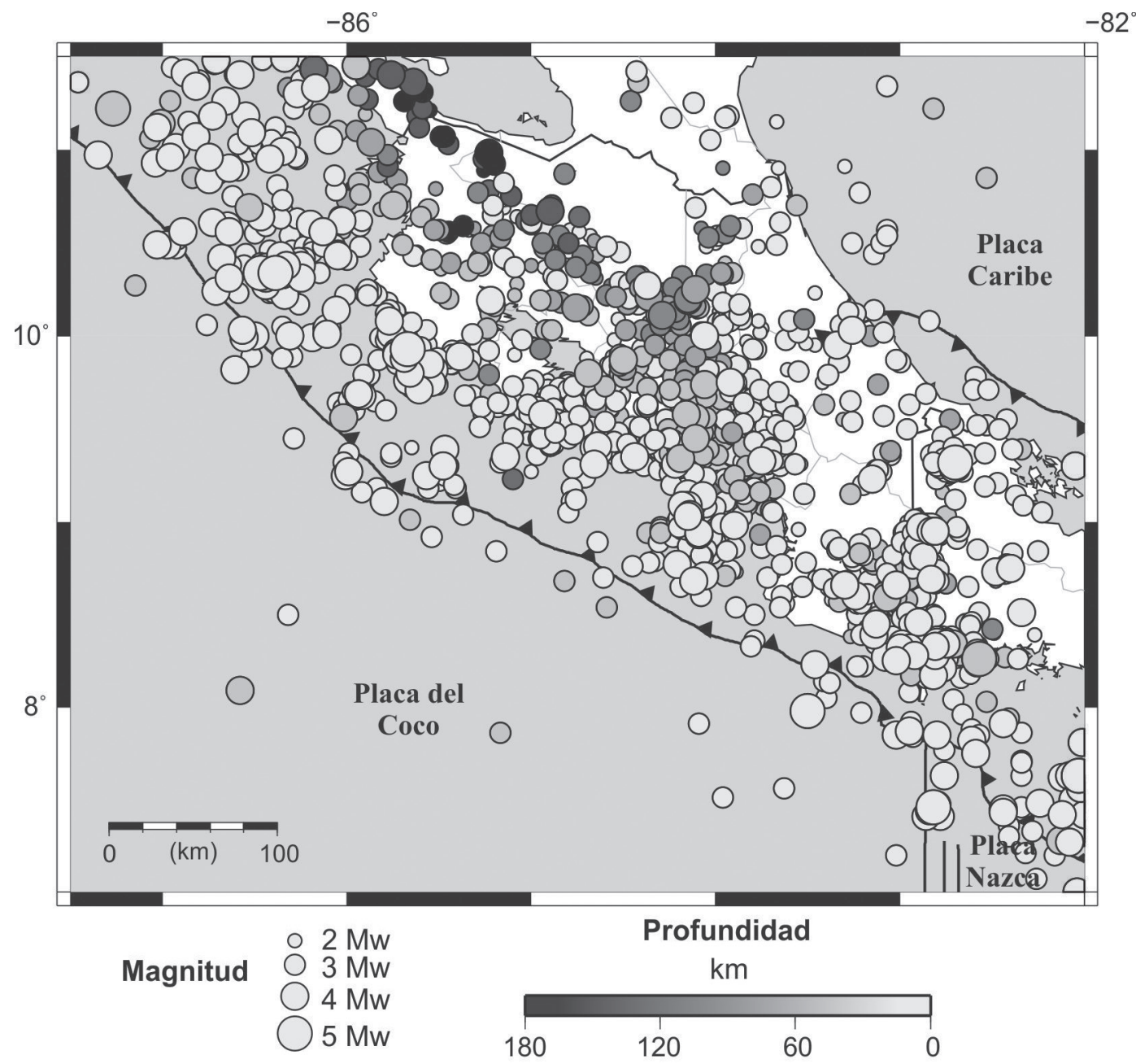

Fig. 1: Ubicación epicentral de los sismos localizados por la RSN durante el año 2013.

Cinco sismos sentidos más tuvieron magnitudes mayores a 5,0 durante el 2013. El más destacado por las altas intensidades generadas fue el ocurrido el 27 de mayo, con una magnitud de 5,6 y ubicado $19 \mathrm{~km}$ al suroeste de Sixaola, en territorio panameño. Este sismo se originó en una falla relacionada con el Cinturón Deformado del Norte de Panamá y fue sentido en todo Costa Rica y el oeste de Panamá.

Otro sismo de magnitud importante ocurrió el 23 de junio, $15 \mathrm{~km}$ al noroeste de Sámara. Este sismo de magnitud 5,5 ocurre en la zona 


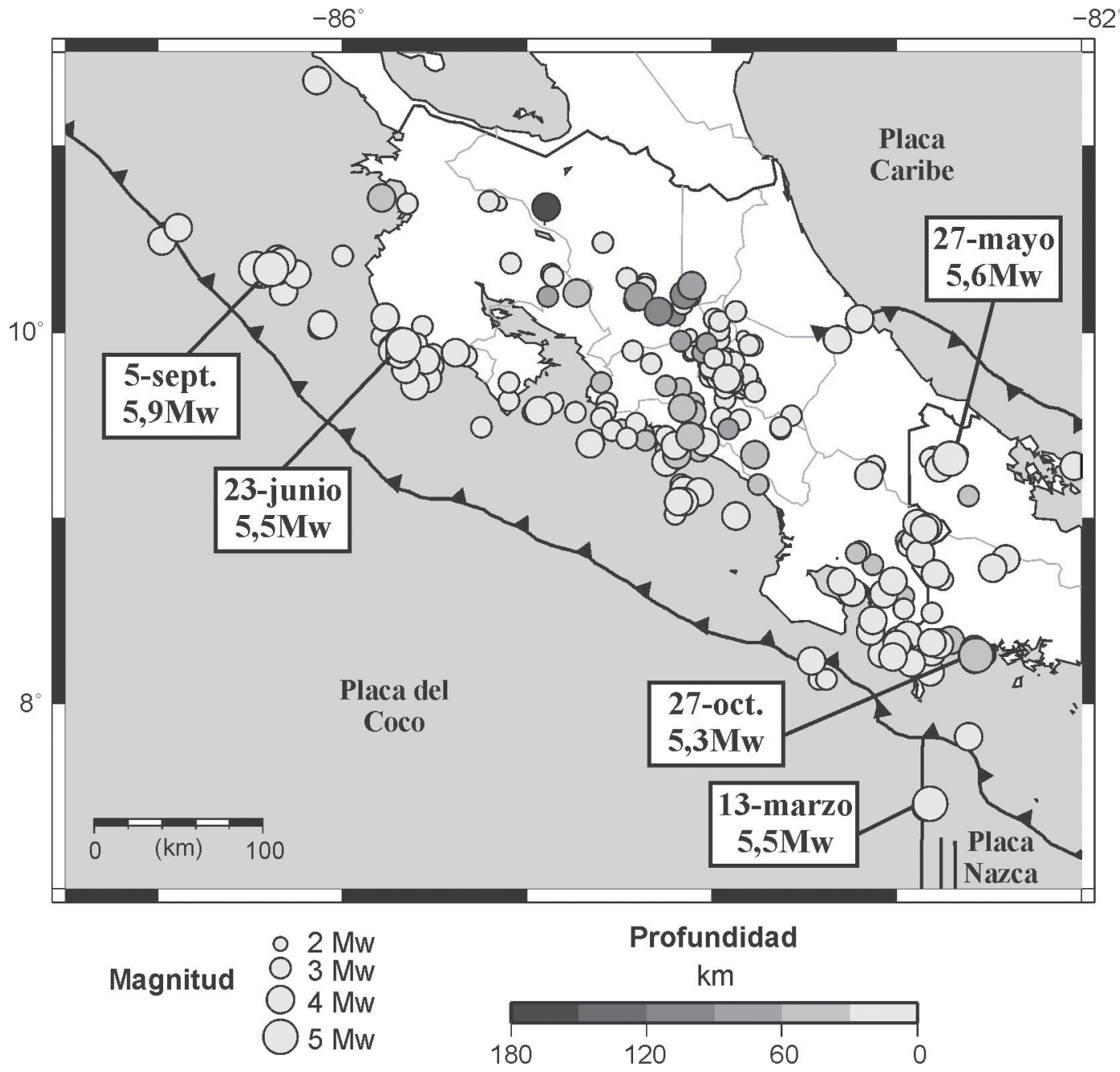

Fig. 2: Ubicación epicentral de los sismos sentidos durante el 2013 en Costa Rica.

de ruptura del terremoto que ocurrió en esa región en el $2012(\mathrm{Mw}$ 7,6) y fue percibido fuerte en toda la península de Nicoya. Dos sismos más de magnitud mayor a 5,0 fueron sentidos de forma moderada en la zona sur. Estos ocurrieron el 13 de marzo y el 27 de octubre, con magnitudes de 5,3 y 5,5 respectivamente, ubicados al sur y sureste de Puerto Armuelles en territorio panameño (Fig. 2).
El 34\% de los sismos sentidos del 2013 tuvieron magnitudes que oscilaron entre 4,0 y 4,9. La mayoría (56\%) de los sismos percibidos tuvieron una magnitud de entre 3,0 y 3,9 (Fig. 3B). El sismo de menor magnitud del 2013 que fue reportado como sentido ocurrió el 22 de agosto $(\mathrm{Mw} 2,5)$ y se localizó $3 \mathrm{~km}$ al norte de Cuipilapa de Bagaces, en Guanacaste. En total, 
20 sismos pequeños $(\mathrm{Mw} \leq 2,9)$ fueron percibidos por la población (Fig. 3B), la mayoría tuvieron sus epicentros en zonas pobladas de San José como Alajuelita y Desamparados y de Cartago, como Orosi y Tobosi de El Guarco.

\section{Distribución por profundidad}

El $85 \%$ de los sismos sentidos tuvieron profundidades menores a $30 \mathrm{~km}$ (Fig. 3C). La mayoría de los sismos tuvieron profundidades menores a 9,9 km (108 sismos) y entre 10 y 19,9 km (74 sismos). Cuarenta sismos sentidos tuvieron su hipocentro a una profundidad mayor de $30 \mathrm{~km}$. Estos sismos están asociados con la deformación interna de la placa del Coco que se subduce debajo de la placa Caribe y la microplaca de Panamá. Los sismos de profundidad intermedia, esto es, de entre $30 \mathrm{~km}$ y $300 \mathrm{~km}$, suelen no ser percibidos por la población a menos que sean de una magnitud intermedia o alta, ya que la energía se atenúa a lo largo del viaje de las ondas sísmicas desde un hipocentro profundo hasta la superficie. El sismo sentido más profundo del 2013 ocurrió el 27 de abril, tuvo una magnitud de 4,2 y se localizó $9 \mathrm{~km}$ al este de San Rafael de Guatuso a una profundidad de $156 \mathrm{~km}$. Otros tres sismos tuvieron una profundidad de entre 91 y $96 \mathrm{~km}$, ocurrieron bajo Varablanca de Heredia (24 de junio, magnitud 4,3 y profundidad $96 \mathrm{~km}$ ), San Roque de Grecia (16 de marzo, magnitud 4,2 y profundidad $92 \mathrm{~km}$ ) y Carrizal de Alajuela (12 de noviembre, magnitud 3,3 y profundidad $91 \mathrm{~km}$ ). El sismo de mayor tamaño de profundidad intermedia ocurrió el 24 de enero, tuvo una magnitud de 4,9 y se localizó en Orotina a una profundidad de $58 \mathrm{~km}$.

\section{Distribución por origen}

La mayoría de los sismos sentidos del 2013 (52\%) fueron originados en fallas locales y regionales (Fig. 3D). Esto incluye los sismos superficiales $(<30 \mathrm{~km})$ ocurridos dentro de la placa
Cuadro 1

Número de sismos localizados y sentidos durante el 2013

\begin{tabular}{ccc}
\hline Mes & Sismos Localizados & Sismos Sentidos \\
\hline Enero & 176 & 30 \\
Febrero & 131 & 21 \\
Marzo & 107 & 25 \\
Abril & 127 & 17 \\
Mayo & 190 & 21 \\
Junio & 299 & 22 \\
Julio & 301 & 9 \\
Agosto & 244 & 31 \\
Setiembre & 226 & 14 \\
Octubre & 238 & 24 \\
Noviembre & 254 & 25 \\
Diciembre & 254 & 22 \\
\hline TOTAL & 2547 & 261 \\
\hline
\end{tabular}

Caribe y la microplaca de Panamá y en las fallas del Cinturón Deformado del Norte de Panamá (Adamek et al., 1988) y Cinturón Deformado del Centro de Costa Rica (Marshall et al., 2000). Otro porcentaje importante de los sismos sentidos (45\%) ocurrió debido al proceso de subducción de la placa del Coco bajo la placa Caribe y la microplaca de Panamá (Fig. 3D). Este proceso incluye los sismos ocurridos en la zona interplacas y los de profundidad intermedia $(>50 \mathrm{~km})$ que se asocian con la deformación interna de la placa del Coco que se subduce bajo Costa Rica. Adicionalmente, siete sismos fueron originados en la Zona de Fractura de Panamá, que corresponde con el límite entre las placas del Coco y Nazca (Fig. 2).

\section{Intensidades}

La intensidades (Escala Mercalli Modificada, MM) más altas observadas durante el 2013 fueron ocasionadas por los sismos del 27 de mayo (Mw 5,6) y del 23 de junio (Mw 5,5), con epicentros cerca de Sixaola y Sámara, respectivamente 

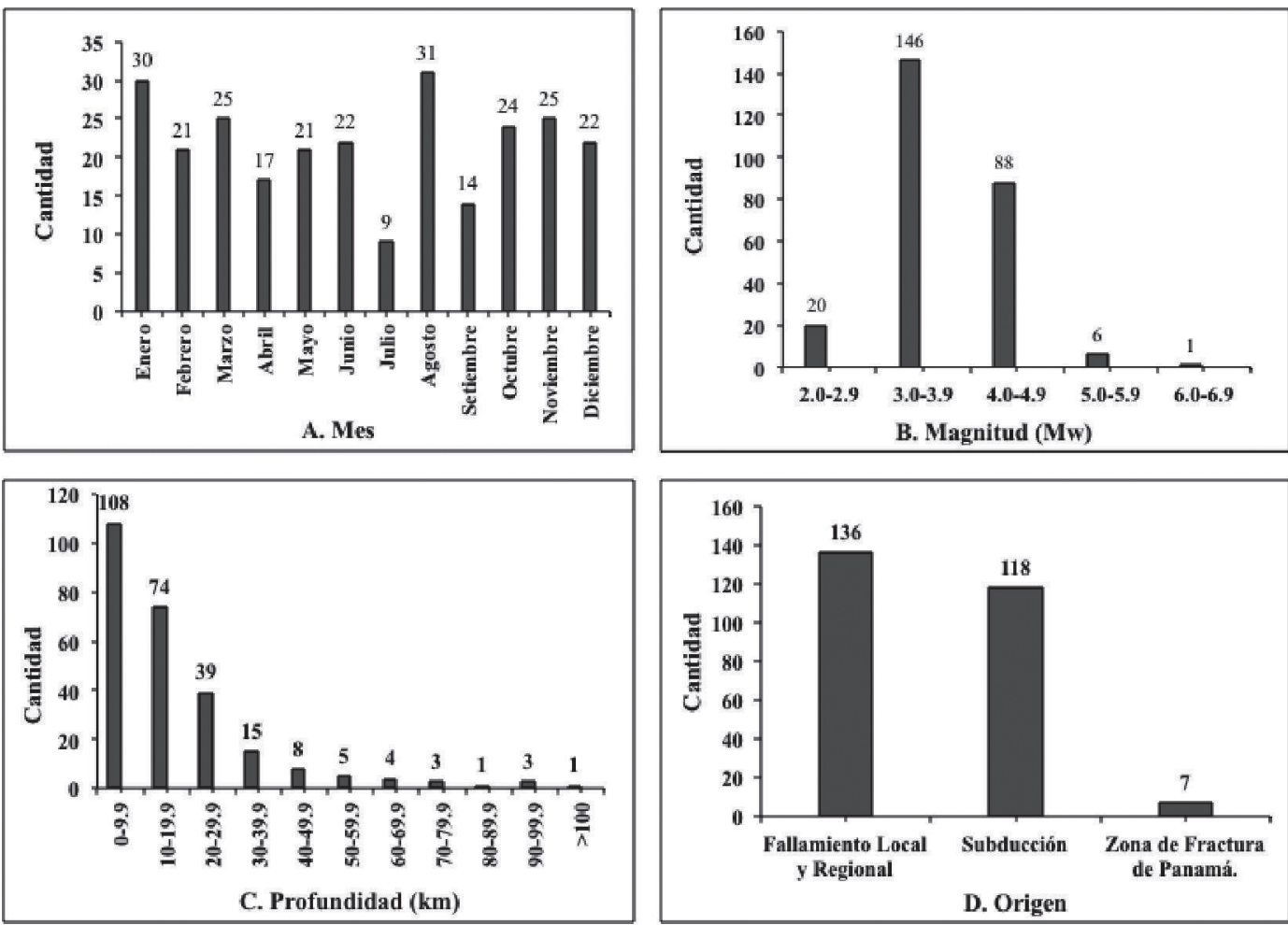

Fig. 3: Distribución de los sismos sentidos del 2013 por A) mes B) magnitud C) profundidad y D) origen. La cantidad de sismos se muestra sobre la barra de cada histograma.

(Fig. 2). Ambos sismos fueron percibidos en todo Costa Rica. En el caso del sismo del 27 de mayo, la zona epicentral experimentó intensidades de VI (MM) con caída de objetos de estanterías en comercios en la zona de Sixaola. La intensidad observada en el Valle Central fue de IV (MM). En el caso del sismo del 23 de junio, se observó una intensidad de V (MM) en la zona de Sámara y Nosara, en la península de Nicoya.

Los otros dos sismos que fueron sentidos en gran parte del territorio de Costa Rica fueron los ocurridos el 24 de enero, cerca de Orotina de magnitud 4,9 y el 2 de octubre cerca de Arancibia en Puntarenas, de magnitud 4,5. Los hipocentros de estos sismos estuvieron ubicados a 56 y $58 \mathrm{~km}$ de profundidad, respectivamente. Ambos sismos fueron percibidos en gran parte de Costa Rica y con una intensidad de hasta IV (MM) en Puntarenas y San Ramón. Además fueron percibidos en localidades del Valle Central y Naranjo, Grecia, Jacó, Parrita, Guápiles, Tres Ríos y Ciudad Quesada.

\section{AGRADECIMIENTOS}

A todo el personal de la RSN en la Escuela Centroamericana de Geología de la Universidad de Costa Rica (UCR) y en el Área de Amenaza y Auscultación Sísmica y Volcánica del Instituto Costarricense de Electricidad (ICE). 
REFERENCIAS BIBLIOGRÁFICAS

ADAMEK, S., FROHLICH, C. \& PENNINGTON, W., 1988: Seismicity of the CaribbeanNazca Boundary Constraints on Microplate Tectonics of the Panama Region.- J. Geophys. Res. 93: 2053-2075.

LIENERT, B. R. \& HAVSKOV, J., 1995: A computer program for locating earthquakes both locally and globally.- Seis. Res. Lett. 66: 26-36, DOI: 10.1785/gssrl.66.5.26
MARSHALL, J. S., FISHER, D. M. \& GARDNER, T. W., 2000: Central Costa Rica deformed belt: Kinematics of diffuse faulting across the western Panama block.- Tectonics, 19: 468492.

OTTEMÖLLER, L., VOSS, P. \& HAVSKOV, J., 2011: SEISAN: Tthe earthquake analysis software for Windows, Solaris, LINUX, and MACOSX, version 9.0.1.- 361 págs. Univ. of Bergen, Bergen. 